\title{
Real-World Data on the Adverse Metabolic Effects of Second-Generation Antipsychotics and Their Potential Determinants in Adult Patients: A Systematic Review of Population-Based Studies
}

\author{
Miquel Bernardo (D) · Fernando Rico-Villademoros • Clemente García-Rizo • \\ Rosa Rojo · Ricardo Gómez-Huelgas
}

Received: January 20, 2021 / Accepted: February 26, 2021 / Published online: April 7, 2021

(C) The Author(s) 2021

\begin{abstract}
Introduction: To assess the risk of occurrence and potential determinants of metabolic disorders in adult patients treated with second-generation antipsychotics (SGAs) under real-world practice conditions.

Methods: MEDLINE, EMBASE, and PsycInfo were searched in July 2019 from database inception. We included population-based, longitudinal, comparative studies that report the results of the outcomes of interest for adult
\end{abstract}

Supplementary Information The online version contains supplementary material available at https:// doi.org/10.1007/s12325-021-01689-8.

M. Bernardo $(\varangle)$ · C. García-Rizo

Department of Psychiatry, Hospital Clínic, University of Barcelona, Idibaps, Cibersam, Barcelona, Spain

e-mail: bernardo@clinic.cat

F. Rico-Villademoros

Institute of Neurosciences, University of Granada, Granada, Spain

F. Rico-Villademoros

Cociente S.L., Madrid, Spain

R. Rojo

Faculty of Health Sciences, Alfonso X El Sabio

University, Villanueva de la Cañada, Madrid, Spain

R. Gómez-Huelgas

Internal Medicine Department, Instituto de

Investigación Biomedica de Malaga-IBIMA, Regional

University Hospital of Malaga, Malaga, Spain participants, including diabetes, ketoacidosis, hyperosmolar hyperglycemic state, weight gain/ obesity, dyslipidemia, hypertension, and metabolic syndrome. Two reviewers independently extracted data on the study design, study quality, and study outcomes.

Results: We included 40 studies. Most studies showed that clozapine and olanzapine were associated with an increased likelihood of developing diabetes, while the results for risperidone and quetiapine were mixed. Although less well studied, ziprasidone and aripiprazole appeared to not be associated with the occurrence of diabetes. Information on antipsychotic-induced weight gain/obesity is extremely scarce. Regarding dyslipidemia, aripiprazole was not associated with an increased likelihood of developing dyslipidemia, clozapine was associated with an increased likelihood of developing dyslipidemia, and risperidone, olanzapine, quetiapine, and ziprasidone showed mixed results. Two studies suggested an association between ziprasidone and the occurrence of hypertension. Several studies found that the occurrence of a metabolic disorder acted as a risk factor for the development of other metabolic disorders. We did not find information on brexpiprazole, cariprazine, or lurasidone, and data on any long-acting SGA were lacking.

Conclusion: Although there are relevant differences among SGAs concerning the risk of metabolic disorders, it appears that none of the 
SGAs included in our review are fully devoid of these disturbances.

\section{PLAIN LANGUAGE SUMMARY}

Patients with severe mental disorders often present metabolic disorders such as obesity, increased blood pressure, high blood sugar, and abnormal cholesterol or triglyceride levels. Treatment with antipsychotics may contribute to the occurrence of these disorders. Using a systematic review, we have evaluated the risk of occurrence of these metabolic disorders associated with the most frequently used antipsychotics-the so-called second-generation antipsychotics (SGAs)-and which factors increase the patient chances of presenting a metabolic disorder when they are treated with these drugs under routine clinical practice. After reviewing 40 studies, we found that, although there are relevant differences among SGAs concerning the risk of metabolic disorders, it appears that none of the drugs included in our review are fully free of these disturbances. Among the factors that increase the chances of these disturbances, we highlight that the presence of a particular metabolic disorder (e.g., increased blood pressure) acts as a risk factor for the occurrence of other metabolic disorders (e.g., high blood sugar), and that the duration of treatment could be a relevant factor for the occurrence of these disorders. Finally, we also found important gaps in our knowledge about this matter, mainly the limited information on the SGAs apparently associated with lower risk of metabolic disorders in experimental studies (that is, few studies evaluating ziprasidone and aripiprazole, and none evaluating brexpiprazole, cariprazine, or lurasidone) and the lack of information on long-acting injectable (that is, antipsychotics that are usually given every 2-4 weeks) SGAs.

Keywords: Antipsychotic;

Diabetes; Dyslipidemia; Hyperosmolar hyperglycemic state; Hypertension; Ketoacidosis; Metabolic syndrome; Obesity; Weight gain

\section{Key Summary Points}

Why carry out this study?

Information on antipsychotic-associated metabolic disorders is derived from shortterm (i.e., 6 weeks) randomized clinical trials-or systematic reviews of these trials-conducted in selected populations devoid of relevant medical or psychiatric comorbidities that received limited concomitant medications

Information from patients treated under real-world conditions for longer time would be useful for better delineating the risk of antipsychotic-associated metabolic disorders and their potential determinants

This systematic review of populationbased case-control and cohort studies aimed to assess the risk of occurrence and potential determinants of metabolic disorders in adult patients treated with second-generation antipsychotics (SGAs) under real-world practice conditions

\section{What was learned from the study?}

Although there are relevant differences among SGAs concerning the risk of metabolic disorders, it appears that none of the SGAs included in our review are fully devoid of these disturbances and there are important gaps in the literature, mainly the limited information on the SGAs apparently associated with better metabolic profile (i.e., few studies evaluating ziprasidone and aripiprazole, and none evaluating brexpiprazole, cariprazine, or lurasidone) and the lack of population-based studies on long-acting injectable SGAs

Among potential determinants of these disturbances, we highlight because of their clinical implications that the presence of a particular metabolic disorder acts as a risk factor for the occurrence of other metabolic disorders, and the length of antipsychotic exposure could be a relevant factor for the occurrence of these disorders 


\section{DIGITAL FEATURES}

This article is published with digital features, including a summary slide and plain language summary, to facilitate understanding of the article. To view digital features for this article go to $\quad$ https://doi.org/10.6084/m9.figshare. 14113586.

\section{INTRODUCTION}

Metabolic disorders, frequently grouped as the so-called metabolic syndrome (i.e., the co-occurrence of abdominal obesity, hyperglycemia, hypertension, and hyperlipidemia), are common in patients with severe mental disorders [1-5] and play a determinant role in the increased risk of premature mortality, predominantly due to cardiovascular diseases, in psychiatric patients [6].

Treatment with antipsychotics may contribute to the occurrence of metabolic syndrome, and thus, clinical practice guidelines recommend initial physical and laboratory assessments and periodic follow-up after initiating antipsychotic treatment for the prevention, early recognition, and treatment of these metabolic disturbances [7]. Results from randomized clinical trials (RCTs) show that there are marked differences among antipsychotics in regard to their risks of inducing metabolic disorders [8]. However, this information is derived from short-term (i.e., 6 weeks) studies conducted in selected populations devoid of relevant medical or psychiatric comorbidities that received limited concomitant medications. Therefore, information from patients treated under real-world conditions for longer time would be useful for better delineating the risk of antipsychotic-associated metabolic disorders.

A systematic review on this topic has been published [9]. However, this otherwise interesting review is limited by the exclusion of studies conducted in subpopulations, such as studies conducted in patients with schizophrenia, patients with bipolar disorder, or hospitalbased studies, and did not include information on the potential moderators of the risk of metabolic disorders. The review included studies published only until July 2015, a time frame that limits information on most recently marketed antipsychotics (e.g., antipsychotics with apparently good metabolic profiles according to pivotal trials, such as brexpiprazole, cariprazine, and lurasidone [8], which obtained US Food and Drug Administration approval in 2015 or later). Antipsychotic treatment selection should be individualized and based on effectiveness and tolerability in addition to patients' preferences. Increasing knowledge of the tolerability profile of wellestablished antipsychotics as well as recently introduced antipsychotics will help achieve individualized antipsychotic treatment selection [10].

The objectives of this systematic review were to assess the risk of occurrence and potential determinants of diabetes or acute diabetes-related complications, weight gain/obesity, hyperlipidemia, hypertension, or metabolic syndrome in adult patients treated with secondgeneration antipsychotics (SGAs) under realworld practice conditions.

\section{METHODS}

\section{Eligibility Criteria}

\section{Type of Studies}

We focused on real-world population-based studies. We included longitudinal comparative studies (i.e., cohort or case-control studies) since cross-sectional studies do not allow for the establishment of a causal relationship.

\section{Types of Participants}

The studies needed to include or separately report the results of the outcomes of interest for adult participants aged 18 years and older. We did not focus on any specific diagnosis; however, to be included, studies had to be conducted in patients with a psychiatric diagnosis, or the psychiatric diagnosis had been included in the multivariate statistical model. The reason for this criterion is that the psychiatric diagnosis may have an independent impact on the risk of metabolic disorders [11]. 


\section{Types of Interventions}

Our interventions of interest were the following SGAs: amisulpride, aripiprazole, asenapine, brexpiprazole, cariprazine, clozapine, iloperidone, lurasidone, olanzapine, paliperidone, quetiapine, risperidone, sertindole, ziprasidone, and zotepine. We included studies reporting data for individual drugs or for grouped SGAs. Information on first-generation antipsychotics (FGAs) was only sought as a comparator to the SGAs. We had no limitations regarding comparators.

\section{Types of Outcome Measures}

The outcomes of interest of the studies were diabetes, ketoacidosis, hyperosmolar hyperglycemic state, weight gain/overweight/obesity, dyslipidemia (hyperlipidemia) or the specific entities hypercholesterolemia or hypertriglyceridemia, hypertension, and metabolic syndrome. We accepted and recorded any definition of these clinical entities, including diagnoses based on any code system (e.g., ICD10) and on exposure to specific treatments (e.g., antihypertensives). We excluded studies conducted on pregnant women.

To be included in this review, articles needed to report the results of at least one of the outcomes of interest.

\section{Search Methods for Study Identification}

The following databases were searched in July 2019 from inception by a medical information specialist (IS-A, see the Acknowledgments): MEDLINE, EMBASE, and PsycInfo. The search strategy is presented in Supplementary Table 1.

\section{Study Selection}

A reviewer with a background in research methodology (FR-V) determined the eligibility of studies by reading the title/abstract of each study identified by the search. The reviewer discarded studies that clearly did not satisfy the inclusion criteria, and complete copies of the remaining studies were obtained. The reviewer assessed all the initially selected complete articles and selected the papers that met the previously described eligibility criteria; doubts regarding whether an article met the criteria were solved by consensus with a second reviewer (RR). If no agreement could be reached, then a third reviewer (MB) was involved in the assessment.

We did not anonymize the studies prior to the assessment.

\section{Data Extraction and Management}

Two reviewers (FR-V and RR) independently extracted data from half of the studies, each using a standard Microsoft Excel form. Both reviewers cross-checked the data extracted by the other reviewer with the source article. In cases of discrepancies, both reviewers discussed the specific difference to reach an agreement prior to entering data into the final tables. If no agreement could be reached, then a third reviewer (MB) was involved in the assessment.

As characteristics of the studies, we recorded information on the first author, year of publication, journal, study design, country, database, time frame, age as a selection criterion or as the mean/median, diagnosis and the tool used to make the diagnosis, type of exposure and its definition, type of control, outcomes evaluated and definition, multivariate model used in statistical analysis and the covariates included in the model, whether the study used propensity scores either for matching and/or adjusting for, and funding source.

After extracting the results of the studies, we recorded information on the size of the source population, length of treatment, duration of the follow-up, outcome evaluated, type of exposure, sample size of exposed/nonexposed or case/controls, summary statistic (e.g., percentage, incidence rate), type effect measure (i.e., odds ratio $[\mathrm{OR}]$, relative risk [RR], or hazard ratio $[\mathrm{HR}])$, point estimate and $95 \%$ confidence interval (CI) of the effect measure, $p$ value, and point estimates and $95 \% \mathrm{CI}$ of the effect measures of the other potential determinants of the occurrence of metabolic disorders in a table. 


\section{Assessment of Study Quality}

The quality of the studies was evaluated by two reviewers (FR-V and RR) using the Newcastle-Ottawa scale, a widely used tool for evaluating the quality of nonrandomized studies in systematic reviews [12]. The Newcastle-Ottawa scale considers three dimensions in the evaluation by using a "star system": the selection of study groups (maximum 4 stars); the comparability of the groups (maximum 2 stars); and the ascertainment of either the exposure or outcome of interest for case-control or cohort studies, respectively (maximum 3 stars). To evaluate comparability, we decided to allocate one star if the study used propensity scores for matching and/or adjusting in the statistical analyses; a second star was allocated if the study explicitly reported controlling for age and gender, either by matching or adjusting in the multivariate model.

Following the same criteria used by other authors [13], a "poor" quality score was reflected as 0 or 1 star(s) in selection, 0 stars in comparability, or 0 or 1 star(s) in outcomes.

\section{Data Synthesis}

Because substantial heterogeneity was expected, no quantitative synthesis was expected to be carried out. The results of individual studies are presented using forest plots for descriptive purposes. Since they require a different interpretation, forest plots are presented separately for odds ratios/relative risks (for binary outcomes) and hazard ratios (for time-to-event outcomes). When reported in the same forest plot, odds ratios and relative risks are clearly identified.

\section{Compliance with Ethics Guidelines}

This article is based on previously conducted studies and does not contain any new studies with human participants or animals performed by any of the authors.

\section{RESULTS}

\section{Study Selection}

Finally, 40 studies published between 2001 and 2019 were included in this review. The flow chart of the study selection process is presented in Fig. 1.

\section{Study Characteristics}

A description of the main characteristics of the studies included in this review is presented in Supplementary Table 2 . Twenty-six $(65.0 \%)$ of the studies had a retrospective cohort design and $14(35.0 \%)$ were case-control studies. Five (12.5\%) studies employed a propensity score methodology, three using matching and two using propensity scores for adjustment in the statistical analysis. Most of the studies were conducted in North America, 24 in the USA and 5 in Canada; 5 studies were conducted in Taiwan; 5 studies were in Europe; and 1 study used databases from the UK and Canada. Nineteen of the studies were partially or fully supported by a pharmaceutical company (12 studies were supported by the comarketer of aripiprazole), 14 received noncommercial support, 6 did not report the funding source, and 1 did not receive any support.

Six (15.0\%) studies included subjects aged 65 or older. Fourteen (35.0\%) studies included patients with schizophrenia, three (7.5\%) patients with bipolar disorder, and two (5.0\%) patients with depression, and the remaining studies included patients with a variety of diagnoses. The antipsychotics most commonly studied were olanzapine $(n=26,65.0 \%)$, risperidone $(n=21,52.5 \%)$, quetiapine $(n=20$, $50.0 \%)$, and clozapine $(n=15,37.5 \%)$, while the less commonly studied antipsychotics were ziprasidone $(n=7,17.5 \%)$ and aripiprazole $(n=5,12.5 \%)$. We did not find any studies that evaluated any other SGAs under study (namely, amisulpride, asenapine, brexpiprazole, cariprazine, iloperidone, lurasidone, paliperidone, sertindole, or zotepine) or data on any longacting SGA. The most common comparators were FGA $(n=17,42.5 \%)$ and patients not 


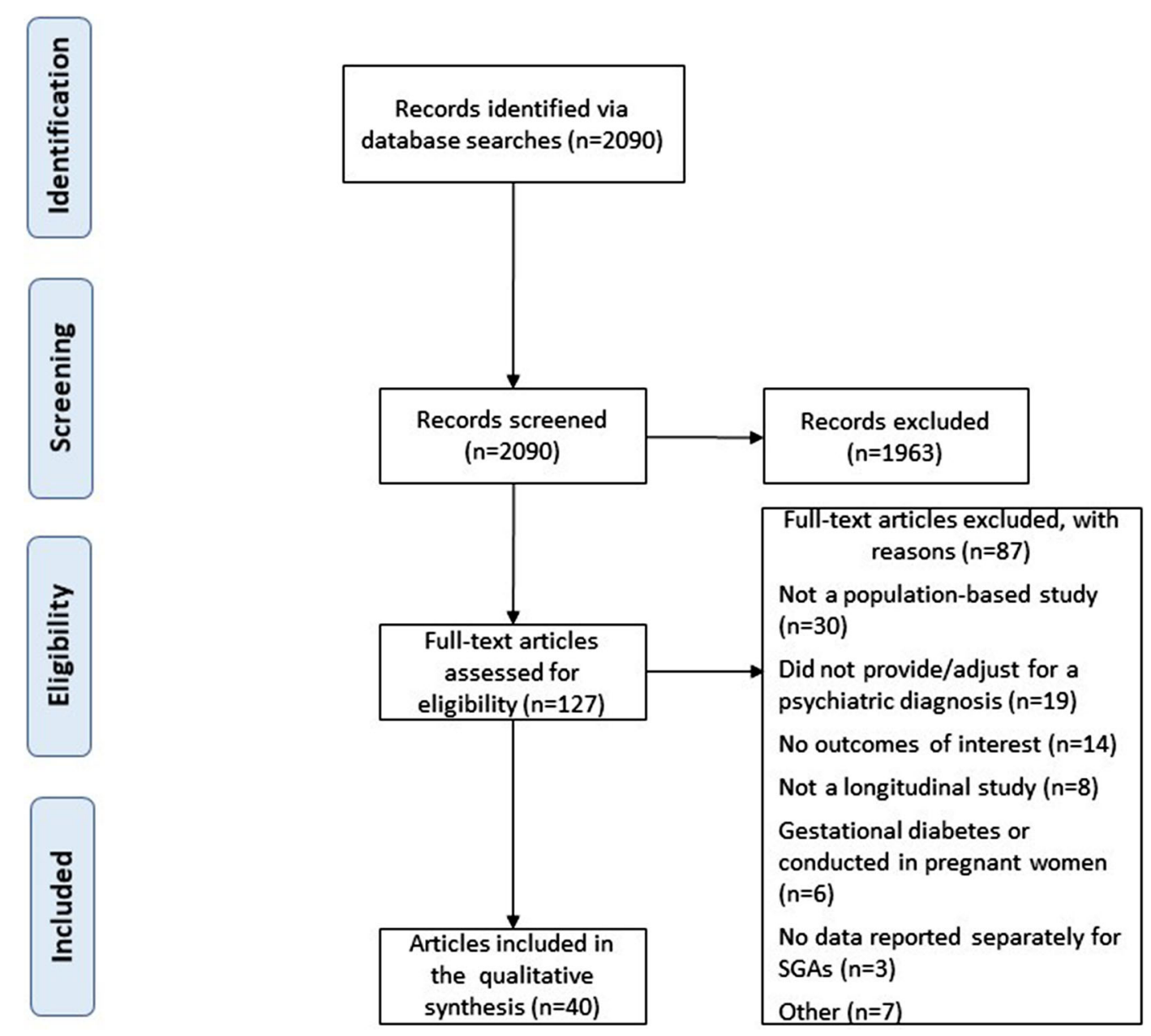

Fig. 1 Flow diagram study selection

receiving antipsychotics ( $n=13,32.5 \%)$. Three studies provided a comparison of the various SGAs. Two studies were conducted with antipsychotic-naïve patients as a comparator, and another study used the general population. Regarding outcomes, diabetes $(n=32,80 \%)$ was the most commonly studied, followed by dyslipidemia $(n=9,22.3 \%)$, hypertension $(n=7$, $17.5 \%)$ hyperglycemia/ketoacidosis $(n=4$, $10.0 \%)$, and weight gain/obesity $(n=3,7.5 \%)$; we did not find any study reporting the risk of occurrence of metabolic syndrome as a whole.

\section{Risk of Bias Within Studies}

The evaluation of the quality of the studies using the Newcastle-Ottawa scale is presented in Supplementary Table 3 for the cohort studies and in Supplementary Table 4 for the case-control studies. Six (23.1\%) of the 26 cohort studies and 1 (7.1\%) case-control study had at least one dimension categorized as poor quality.

\section{Results of Individual Studies}

\section{Studies Conducted in Adults}

Diabetes The results of the studies comparing the risk of occurrence of diabetes with individual antipsychotics compared to subjects who did not receive antipsychotics are presented in Fig. 2a. Two studies showed that clozapine is associated with an increased likelihood of occurrence of diabetes, with heterogeneous 


\begin{tabular}{|c|c|c|}
\hline Author, Year & Exposure & Control \\
\hline Gianfrancesco, 2002 & Clo & Without APS \\
\hline Gianfrancesco (Ohio Medicaid), 2006 & Clo & Without APS \\
\hline Gianfrancesco, 2002 & Ris & Without APS \\
\hline Koro, 2002 & Ris & Without APS \\
\hline Gianfrancesco, 2003 & Ris & Without APS \\
\hline Gianfrancesco (Ohio Medicaid), 2006 & Ris & Without APS \\
\hline Gianfrancesco (PharmaMetrix), 2006 & Ris (All) & Without APS \\
\hline Gianfrancesco (PharmaMetrix), 2006 & Ris (Lowa) & Without APS \\
\hline Gianfrancesco (PharmaMetrix), 2006 & Ris (Mediumª) & Without APS \\
\hline Gianfrancesco (PharmaMetrix), 2006 & Ris $\left(\mathrm{High}^{a}\right)$ & Without APS \\
\hline Gianfrancesco, 2002 & Ola & Without APS \\
\hline Koro, 2002 & Ola & Without APS \\
\hline Gianfrancesco, 2003 & Ola & Without APS \\
\hline Gianfrancesco (Ohio Medicaid), 2006 & Ola & Without APS \\
\hline Gianfrancesco (PharmaMetrix), 2006 & Ola (All) & Without APS \\
\hline Gianfrancesco (PharmaMetrix), 2006 & Ola (Lowa) & Without APS \\
\hline Gianfrancesco (PharmaMetrix), 2006 & Ola (Mediumán) & Without APS \\
\hline Gianfrancesco (PharmaMetrix), 2006 & Ola $\left(\mathrm{High}^{\mathrm{a}}\right)$ & Without APS \\
\hline Gianfrancesco, 2003 & Que & Without APS \\
\hline Gianfrancesco (Ohio Medicaid), 2006 & Que & Without APS \\
\hline Gianfrancesco (PharmaMetrix), 2006 & Que (All) & Without APS \\
\hline Gianfrancesco (PharmaMetrix), 2006 & Que (Lowa) & Without APS \\
\hline Gianfrancesco (PharmaMetrix), 2006 & Que (Medium ${ }^{\mathrm{a}}$ ) & Without APS \\
\hline Gianfrancesco (PharmaMetrix), 2006 & Que $\left(\right.$ High $\left.^{a}\right)$ & Without APS \\
\hline Gianfrancesco (Ohio Medicaid), 2006 & Zip & Without APS \\
\hline Kornegay, 2002 & SGAS & Without APS \\
\hline
\end{tabular}

b
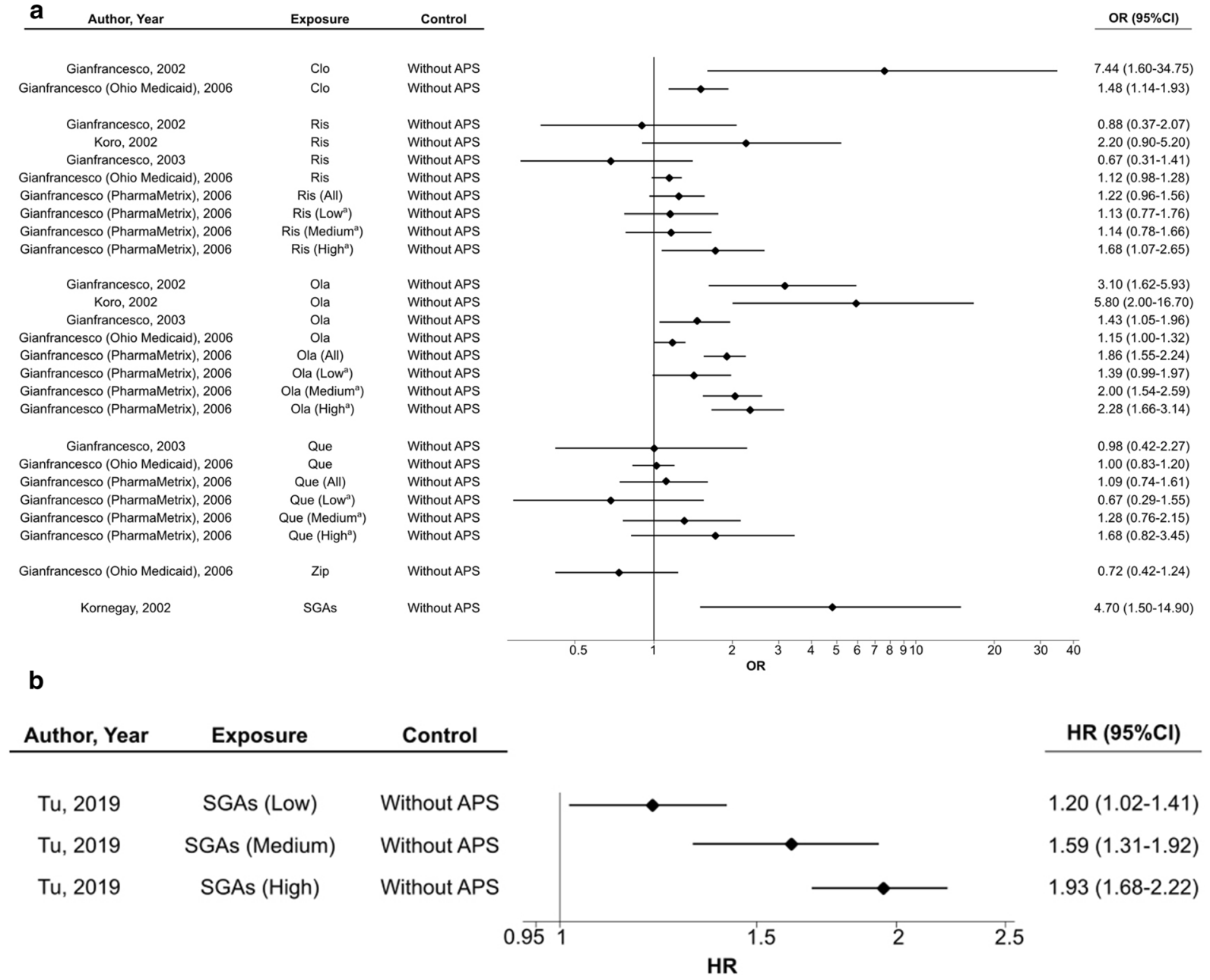

Fig. 2 a Risk of diabetes in adults who received secondgeneration antipsychotics compared to subjects who did

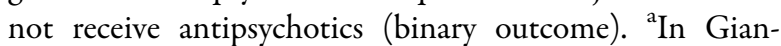
francesco et al. [16] low, medium, and high dose corresponded to the bottom, middle, and top third of the daily dose range for each patient subgroup within each antipsychotic category. b Risk of diabetes in adults who received second-generation antipsychotics compared to subjects who did not receive antipsychotics (time-to-event outcome). Results are reported for young adults (i.e.,
18-29 years) with low exposure (30-179 cumulative defined daily dose [cDDDs]), medium exposure (180-364 cDDDs), and high exposure (at least 365 cDDDs) compared to subjects who did not receive antipsychotics (exposure less than 30 cDDDs). APS antipsychotic, $C I$ confidence interval, $C l o$ clozapine, $H R$ hazard ratio, Ola olanzapine, $O R$ odds ratio, Que quetiapine, Ris risperidone, $S G A s$ second-generation antipsychotics, $Z i p$ ziprasidone

associated with the occurrence of diabetes (OR 1.68, 95\% CI 1.07-2.65) [16]. With heterogeneous results regarding the strength of the association, olanzapine was consistently associated with an increased likelihood of diabetes [14-18]; in one study, the higher the dose, the greater the likelihood of developing diabetes with olanzapine [16]. Compared with subjects 
who did not receive antipsychotics, quetiapine was not associated with the occurrence of diabetes [15-17], regardless of the dose [16]. A single study evaluating ziprasidone did not find an association with the occurrence of diabetes. Compared to subjects who did not receive an antipsychotic, SGAs as a whole were associated with an increased likelihood of diabetes [19] (Fig. 2a), and the likelihood increased as exposure increased [20] (Fig. 2b).

The results of the comparisons of SGAs with FGAs are presented in Fig. 3a for binary outcomes and Fig. $3 \mathrm{~b}$ for time-to-event outcomes. Seven studies reported binary outcomes $[11,18,21-25]$, showing a lack of association with the occurrence of diabetes for risperidone and quetiapine compared to FGA and mixed results for clozapine and olanzapine (Fig. 3a). A study showed no apparent dose-response association between exposure to low, medium, and high doses of clozapine, risperidone, and quetiapine [24]; low doses of olanzapine were not associated with an increased likelihood of developing diabetes (OR 1.10, 95\% CI 0.90-1.50), while medium (OR 1.60, 95\% CI 1.30-1.90) and high doses (OR 1.50, 95\% CI 1.20-1.80) were associated with an increased likelihood of developing diabetes [24]. Comparisons with FGAs using time-to-event outcomes (Fig. 3b) [26-31] showed mixed results for clozapine, risperidone, and quetiapine, while the results for ziprasidone from three studies showed no significantly increased likelihood of developing diabetes; of the six studies, five showed an association between exposure to olanzapine and the likelihood of developing diabetes with heterogeneous results regarding the strength of the association (the HR varied from 1.15 to 3.66 ).

Three studies provided head-to-head comparisons among SGAs and two of them using time-to-event outcomes showed no difference among them for the risk of developing diabetes (Supplementary Fig. 1a) [32, 33], while a study using a binary outcome showed a $20 \%$ increased likelihood of the occurrence of diabetes in olanzapine-treated patients compared to those treated with risperidone (OR 1.20, 95\% CI 1.00-1.43) [34] (Supplementary Fig. 1b). Studies of the risk of developing diabetes in those who
Fig. 3 a Risk of diabetes in adults who received secondgeneration antipsychotics compared to those who received first-generation antipsychotics (binary outcome). Lambert et al. [24] defined alow dose as $<7.5 \mathrm{mg}$ for olanzapine, $<3 \mathrm{mg}$ for risperidone, $<300 \mathrm{mg}$ for clozapine, and $<250$ for quetiapine; ${ }^{b}$ medium dose as $7.5-12.5 \mathrm{mg}$ for olanzapine, 3-6 $\mathrm{mg}$ for risperidone; 300-600 $\mathrm{mg}$ for clozapine; and 250-500 mg for quetiapine; and 'high dose as $<12.5 \mathrm{mg}$ for olanzapine, $>6 \mathrm{mg}$ for risperidone, $>600 \mathrm{mg}$ for clozapine, and $>500 \mathrm{mg}$ for quetiapine. $\mathbf{b}$ Risk of diabetes in adults who received secondgeneration antipsychotics compared to those who received first-generation antipsychotics (time-to-event outcomes). Ari aripiprazole, CI confidence interval, Clo clozapine, $F G A s$ first-generation antipsychotics, $H R$ hazard ratio, Ola olanzapine, $O R$ odds ratio, Que quetiapine, $R$ is risperidone, $R R$ relative risk, $Z$ ip ziprasidone

received SGAs using comparators other than not receiving antipsychotics, FGAs, or head-tohead comparisons are presented in Supplementary Fig. 2 for binary outcomes and Supplementary Fig. 3 for time-to-event outcomes [35-40]. Among these latter studies, Rajkumar et al. [39], in young adults with schizophrenia, showed that the three individual antipsychotics analyzed, namely, clozapine, olanzapine, and aripiprazole, were associated with a significantly increased likelihood of developing diabetes compared with antipsychotic-naïve patients. Nielsen et al. [38] also used antipsychotic-naïve patients as comparators and reported an increased likelihood of developing diabetes for clozapine and olanzapine but a reduced likelihood of developing diabetes for aripiprazole; they reported no association for risperidone, quetiapine, and ziprasidone.

Diabetes-Related Complications Two studies evaluated the risk of developing diabetes-related complications, such as hyperglycemia crisis, ketoacidosis, and a hyperosmolar hyperglycemic state $[29,41]$. One of the studies excluded patients with a previous diagnosis of diabetes [29]. The other study did not exclude patients with diabetes, but most patients did not have a diagnosis of diabetes [41]. These studies showed an increased likelihood of these complications with clozapine (HR 3.75, 95\% CI 
a

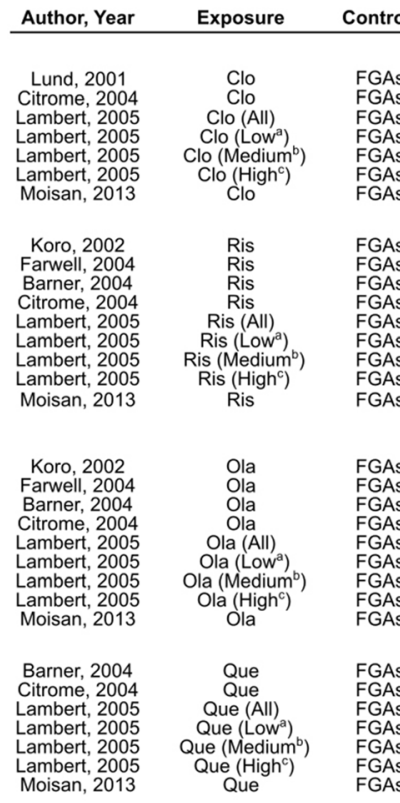

\section{trol}

\section{ass}

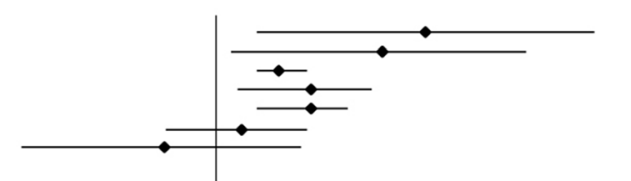

OR $(95 \% \mathrm{Cl})$

RR (95\%Cl)

$2.06(1.07-3.99)$
$1.30(1.20-1.50)$
$1.50(1.10-2.00)$
$1.50(1.20-1.80)$
$1.10(0.80-1.50)$
$0.78(0.42-1.46)$

$1.60(0.70-3.80)$

$0.70(0.40-1.40$

$0.93(0.54-1.58)$
$1.50(0.81-2.79)$

$1.00(0.90-1.20$

$0.90(0.80-1.10)$

$1.00(0.80-1.30)$

$0.99(0.90-1.10)$

$2.50(1.20-5.40)$

(1)

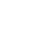

(1.20-5.40)

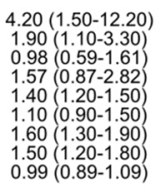

$1.55(0.53-2.49)$

$3.09(1.59-6.03)$

$1.20(0.80-1.70)$

$1.20(0.60-2.40$

$1.60(0.70-3.60)$
$0.89(0.81-0.99)$

\section{b}

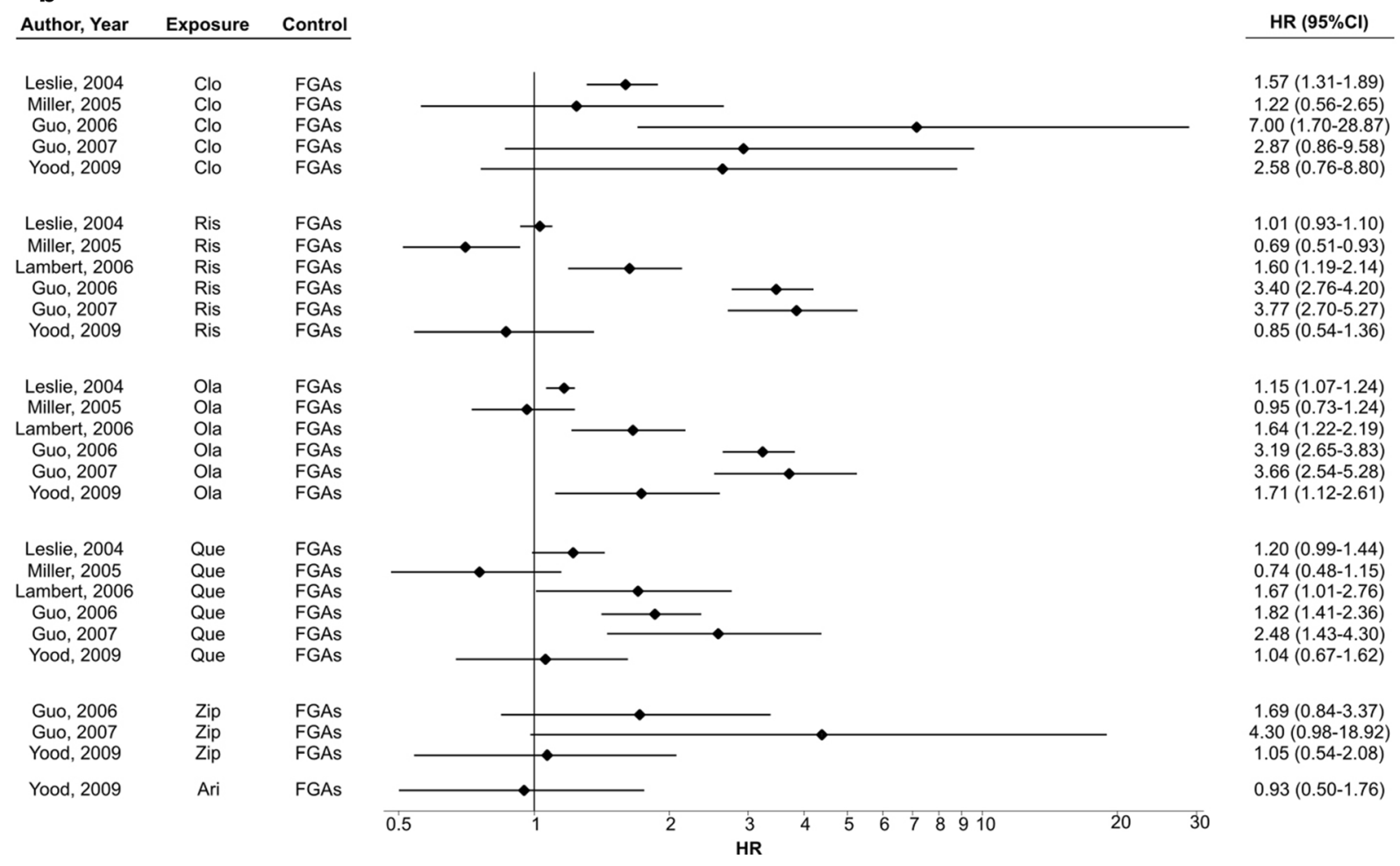


1.39-10.09) and olanzapine (HR 1.77, 95\% CI 1.05-2.98) compared to FGAs and no difference between olanzapine and risperidone or between quetiapine and risperidone in this regard (Fig. 4).

Obesity and Weight Gain We found a single study that evaluated the risk of occurrence of obesity with SGAs, reporting that, compared to FGAs, risperidone, quetiapine, ziprasidone, and aripiprazole were not associated with the occurrence of obesity (the effect sizes were not reported), while olanzapine was associated with a reduced likelihood of occurrence of obesity (OR 0.60, 95\% CI 0.36-1.00) [42].

Two studies reported the risk of weight gain with olanzapine and risperidone compared to the risk with FGAs [23] (Supplementary Fig. 4) and the risk with olanzapine and quetiapine compared to lithium [35] (Supplementary Fig. 5). All three SGAs were associated with an increased likelihood of weight gain compared with the controls.

Dyslipidemia Seven studies reported the risk of developing dyslipidemia with SGAs in adults (Fig. 5a, b) [11, 36, 40, 43-46]. The results of two studies indicated that aripiprazole was not associated with an increased likelihood of developing dyslipidemia, while clozapine was consistently associated with the occurrence of dyslipidemia in three studies. Risperidone, olanzapine, quetiapine, and ziprasidone showed mixed results. Overall, SGAs were associated with an increased risk of developing dyslipidemia compared to patients without schizophrenia [36] and the general population [43] but not compared to FGAs [43, 46].

Hypertension The results of the six studies that evaluated the potential association between treatment with SGAs and the risk of hypertension $[11,35,36,42,46,47]$ are presented in Fig. 6a, b. Two studies conducted by the same research group reported an increased risk of occurrence of hypertension with ziprasidone compared to FGAs; in those studies, the authors reported no association with hypertension for risperidone, olanzapine, quetiapine, and aripiprazole [42, 46]. In another study, compared with lithium, olanzapine was associated with a higher likelihood of developing hypertension, but quetiapine was not [35].

\section{Studies Conducted in Patients Aged 65 Years or Older}

In this review, six studies were conducted specifically in patients aged 65 years or older $[25,48-52]$, and one study reported separate results for patients within this age stratum [41]. The results regarding the risk of occurrence of diabetes are mixed but consistently show that SGAs as a whole are associated with an

\begin{tabular}{cccc} 
Author, Year & Outcome & Exposure & Control \\
\hline Leslie, 2004 & Ketoacidosis & Clo & FGAs \\
Leslie, 2004 & Ketoacidosis & Ola & FGAs \\
& & & \\
Lipscombe, 2014 & Hyperglycemia & Ola & Ris \\
Lipscombe, 2014 & Hyperglycemia & Que & Ris
\end{tabular}

Fig. 4 Risk of hyperglycemia-related complications in individuals who received second-generation antipsychotics (time-to-event outcomes). CI confidence interval, Clo clozapine, FGAs first-generation antipsychotics, $H R$ hazard ratio, Ola olanzapine, Que quetiapine, Ris risperidone.

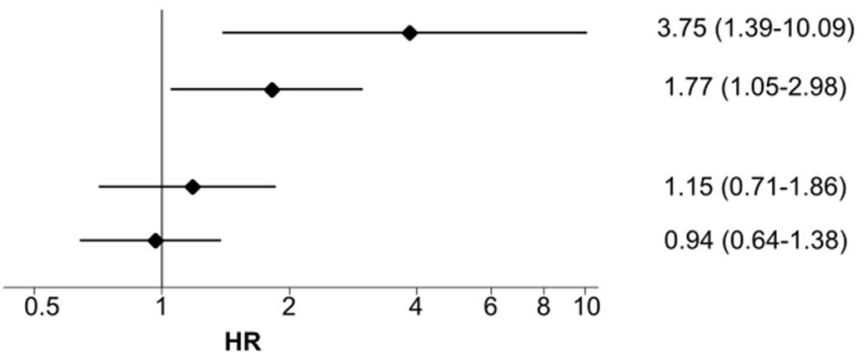

${ }^{a}$ Hyperglycemia was defined as the first hospital admission in the 365 days following drug initiation that was associated with a preadmission diagnosis of hyperglycemia, diabetic ketoacidosis, or a hyperglycemic hyperosmolar state based on ICD-9 and ICD-10 diagnostic codes 


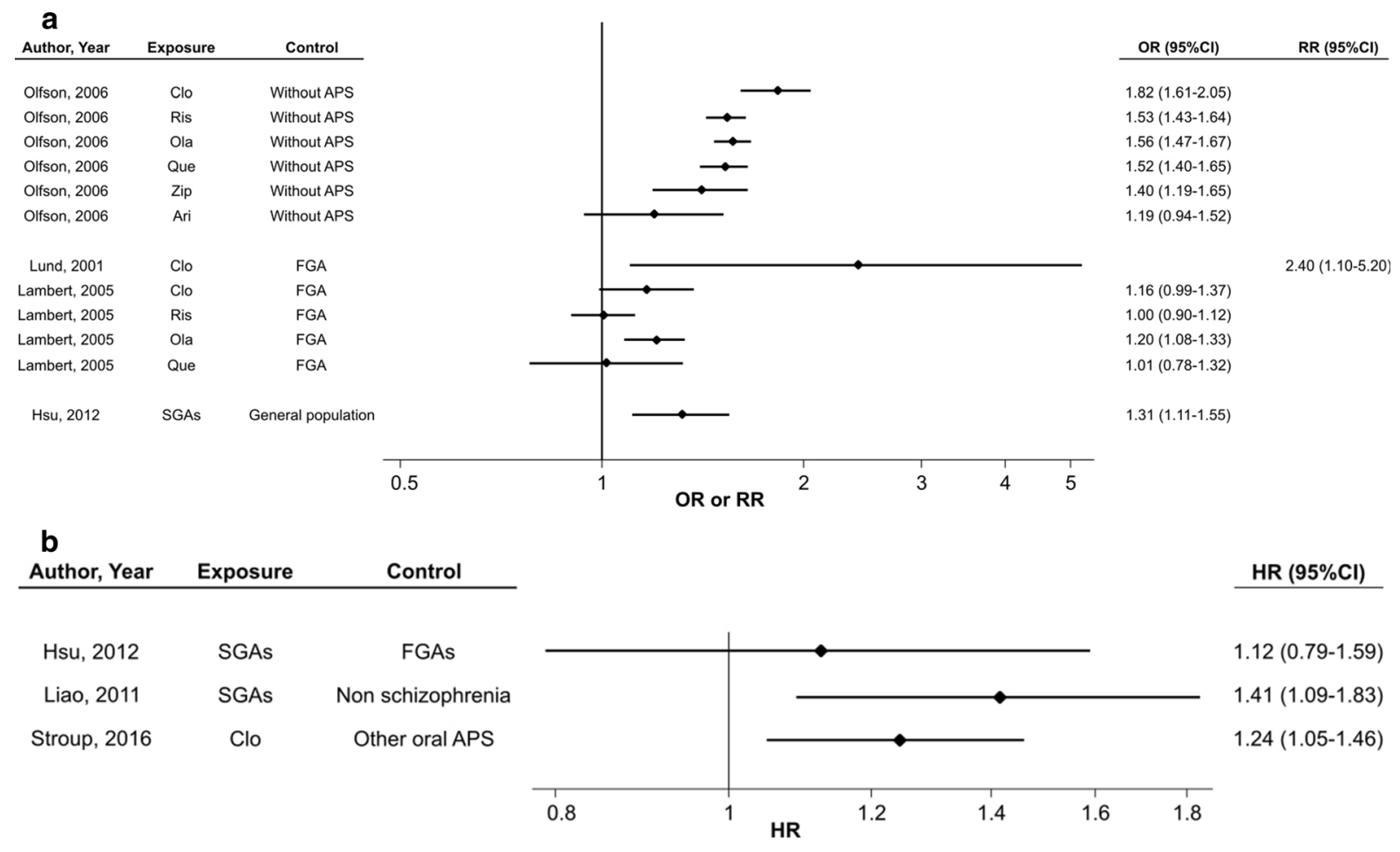

Fig. 5 a Risk of dyslipidemia in individuals who received second-generation antipsychotics (binary outcomes). Note: a study conducted by Jerrell et al. [46] reported that risperidone, olanzapine, quetiapine, ziprasidone, and aripiprazole were not associated with an increased likelihood of dyslipidemia, but the authors did not report the specific results for these SGAs. b Risk of dyslipidemia in

increased likelihood of occurrence of diabetesrelated complications (Fig. 7). One study evaluated the head-to-head risk of metabolic disorders in this population [41], showing that quetiapine was associated with a lower likelihood of developing diabetes-related complications than risperidone (HR 0.69, 95\% CI 0.53-0.90), and olanzapine and risperidone did not differ in terms of the risk of developing diabetes-related complications (HR 1.13, 95\% CI 0.87-1.48) (Fig. 8).

\section{Polypharmacy with Antipsychotics}

Nine studies explicitly stated that they took into consideration the use of a combination of antipsychotics and five of them reported specific results for that subgroup (Supplementary Table 1). Citrome et al. found that, compared to individuals who received second-generation antipsychotics (time-to-event outcomes). APS antipsychotic, Ari aripiprazole, CI confidence interval, Clo clozapine, FGAs firstgeneration antipsychotics, $H R$ hazard ratio, Ola olanzapine, $O R$ odds ratio, Que quetiapine, $R$ is risperidone, $R R$ relative risk, $S G A$ s second-generation antipsychotics, $Z$ ip ziprasidone

FGAs, the use of two or more SGAs was associated with an increased likelihood of occurrence of diabetes among psychiatric inpatients (OR 2.86, 95\% CI 1.57-5.2) [22], and Lambert et al. found that any combination of SGAs and FGAs was associated with an increased likelihood of occurrence of diabetes in patients with schizophrenia (OR 1.6, 95\% CI 1.3-1.9) [24]. However, three additional studies did not find an association between antipsychotic polytherapy and the occurrence of diabetes compared to untreated patients $[14,17]$ or patients receiving FGAs [25].

\section{Potential Determinants of Occurrence of Metabolic Disorders}

Twenty studies reported the results of potential determinants other than the specific 


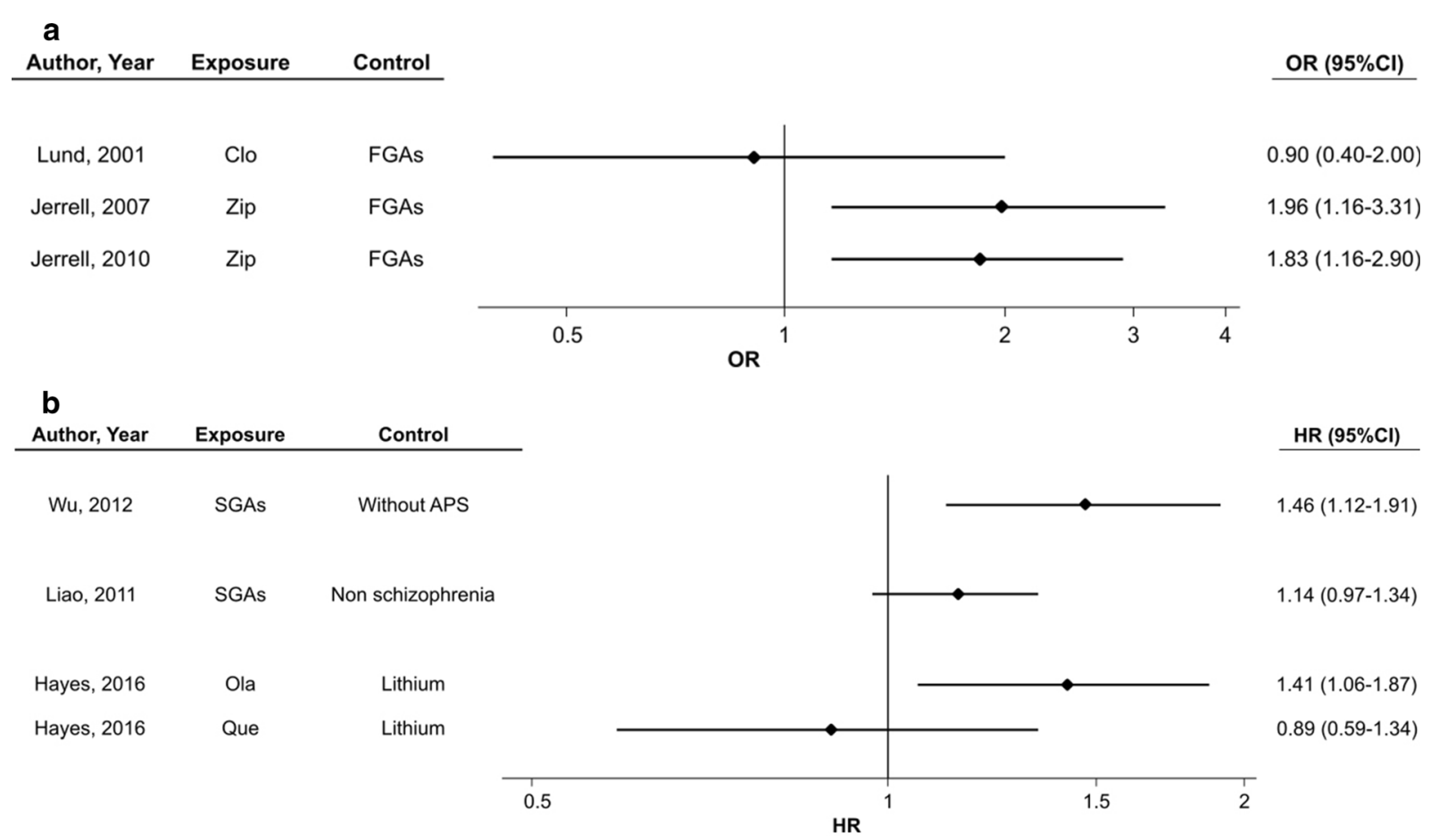

Fig. 6 a Risk of hypertension in individuals who received second-generation antipsychotics (binary outcomes). Jerrell and McIntyre [46] reported that risperidone, olanzapine, quetiapine, and aripiprazole were not associated with an increased likelihood of hypertension, but they did not report the specific results for these SGAs. b Risk of

antipsychotic of the occurrence of diabetes (Supplementary Table 5) [14-17, 19-28, 30, $31,33,36-38]$. The factors most frequently reported as being associated with the occurrence of diabetes were age [14-16, 21, 36-38], nonwhite race $[15,21,24]$, length of exposure $[16,17,25,33]$, obesity/body mass index (BMI) $[15,16,19,20,23,26,27,31]$, hypertension $[19,20,26,27, \quad 36]$, dyslipidemia $[20,21,26,36,37]$, and treatment with concomitant medications, such as beta-blockers $[15-17,24,31]$; thiazides $[15,16,24,31]$; angiotensin-converting enzyme inhibitors [24]; antihypertensives [38]; lipid-lowering drugs [38]; specific lipid-lowering drugs, including fibrates [31] and statins [15, 31]; and selective serotonin reuptake inhibitors (SSRIs) [15, 24]. Regarding the type of psychiatric diagnosis, some studies found an increased risk of occurrence of diabetes for those with schizophrenia hypertension in individuals who received second-generation antipsychotics (time-to-event outcomes). APS antipsychotic, $C I$ confidence interval, Clo clozapine, FGAs firstgeneration antipsychotics, $H R$ hazard ratio, Ola olanzapine, $O R$ odds ratio, Que quetiapine, $S G A s$ second-generation antipsychotics, $Z i p$ ziprasidone

$[16,30,31]$, bipolar disorder [30], and posttraumatic stress disorder [30], while others found that major affective disorders were associated with a reduced risk of occurrence of diabetes [17]; finally, some studies did not find that the type of diagnosis was a factor associated with the occurrence of diabetes [14, 20, 23]. When psychiatric diagnosis was evaluated as a comorbidity, an increased risk of diabetes was reported for impulse control disorders [26] and substance use disorders $[15,27]$, while the presence of a comorbid bipolar disorder was associated with a reduced risk of diabetes in one study [33]. We did not find information on the potential determinants of hyperglycemia complications; however, in a bivariate analysis, the incidence of these complications was higher in patients aged 66 years or older than in those aged 18-65 years, overall and for any antipsychotic [41]. Regarding weight gain, Farwell et al. 


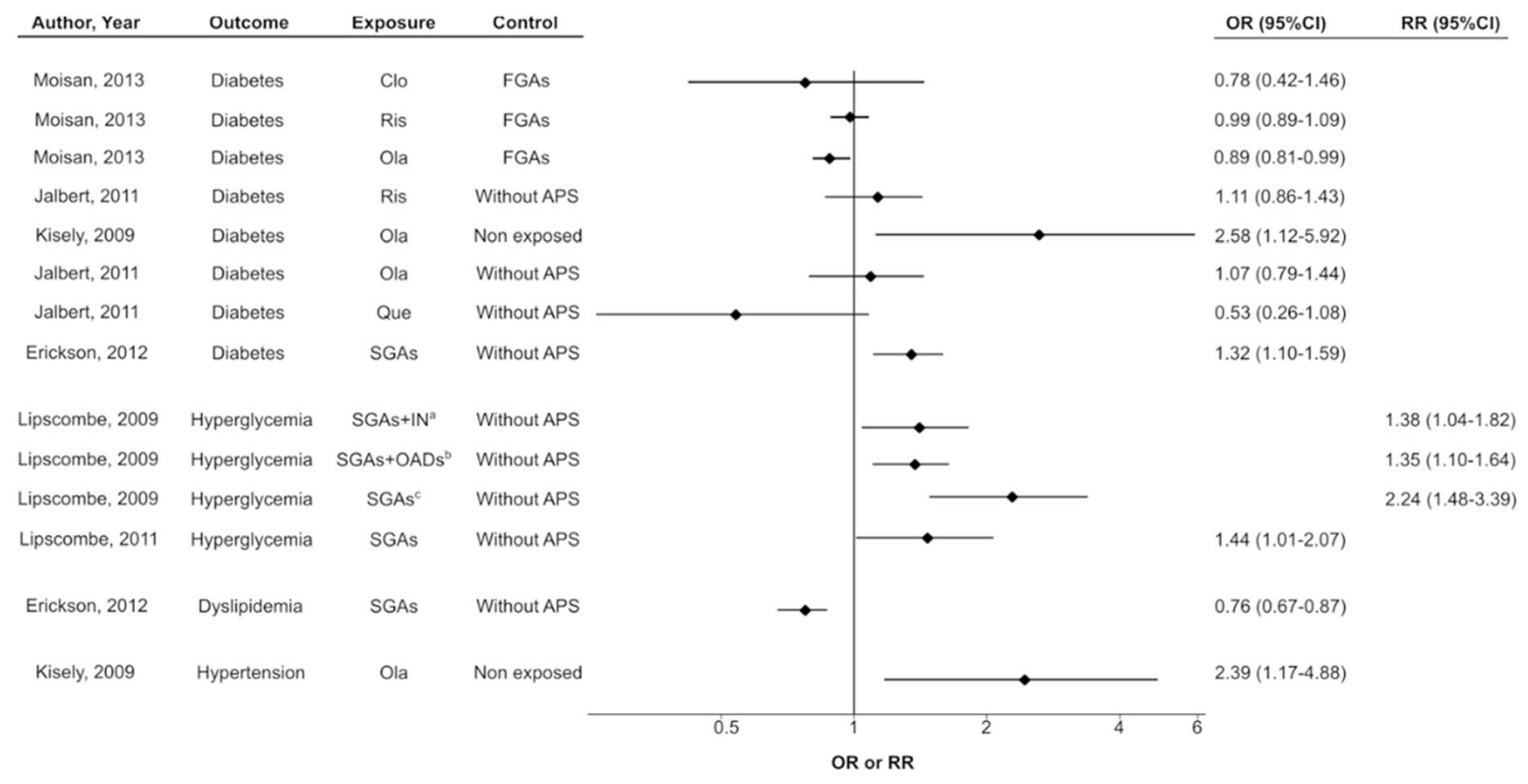

Fig. 7 Risk of metabolic disorders in patients aged 65 years or older who received second-generation antipsychotics (binary outcomes). Lipscombe et al. [51] reported results separately for patients treated with insulin (a), patients who received oral antidiabetic agents $(\mathbf{b})$, and patients who did not receive antidiabetics (c). APS antipsychotic, $C I$ confidence interval, $I N$ insulin, $O A D s$ oral antidiabetic drugs, Ola olanzapine, $O R$ odds ratio, Que quetiapine, $R i$ s risperidone, $R R$ relative risk, $S G A s$ secondgeneration antipsychotics

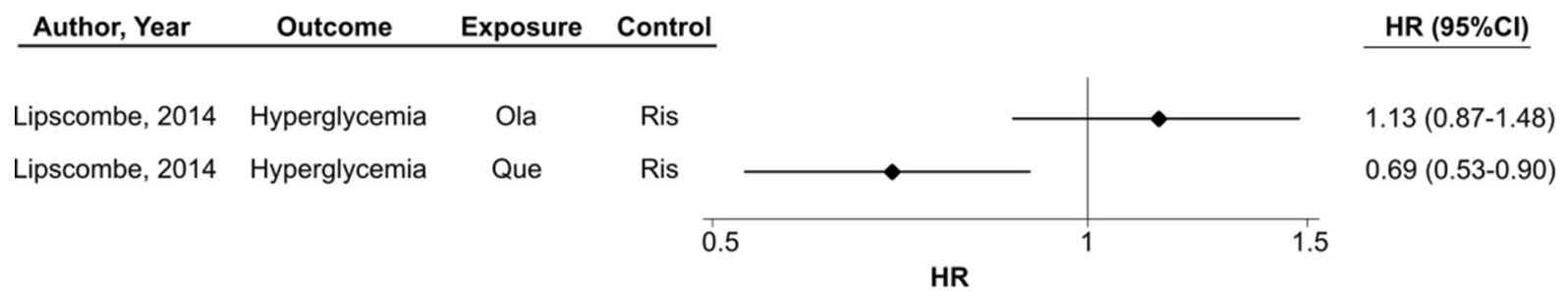

Fig. 8 Risk of metabolic disorders in patients aged 65 years or older who received second-generation antipsychotics (time-to-event outcomes). CI confidence interval,

[23] reported that baseline weight was associated with a reduced risk of weight gain of at least 7\% (OR 0.99, 95\% CI 0.96-0.99).

The potential determinants of the occurrence of dyslipidemia were reported in three studies (Supplementary Table 6) [36, 43, 44]. Age [36, 43], the presence of diabetes $[36,43,44]$, and the presence of hypertension $[36,43]$ were reported to be associated with an increased likelihood of developing dyslipidemia.

Studies reporting the potential determinants of the occurrence of hypertension are shown in
Clo clozapine, $H R$ hazard ratio, Ola olanzapine, $O R$ odds ratio, Que quetiapine, Ris risperidone

Supplementary Table 7. Regarding the potential determinants of the occurrence of hypertension, two studies reported that it increased as age increased [36, 47], and the results regarding sex were conflicting, with one study reporting an increased likelihood of developing hypertension in women (OR 1.38, 95\% CI 1.09-1.74) [42] and another study reporting an increased likelihood in men (HR 1.24, 95\% CI 1.01-1.53) [47]. One study reported that the presence of diabetes or dyslipidemia was associated with an increased likelihood of hypertension [36]. 


\section{DISCUSSION}

Randomized controlled trials provide robust evidence of the efficacy of antipsychotics and important information on their tolerability and safety. However, they require selected samples for isolating the drug effects [53]. In addition, these explanatory trials, usually performed for registrational purposes, are focused on the efficacy of drugs and with short-term duration. Therefore, they are not the best option for evaluating infrequent outcomes (e.g., diabetic ketoacidosis) or those that require longer latency periods such it is the case of most metabolic side effects. Therefore, this information should be complemented with the results of long-term-preferably pragmatic-trials or observational studies that better resemble clinical practice.

According to our review, diabetes is the most frequently studied metabolic disorder under real-world conditions using a population-based design; this is consistent with the long-standing interest in the relationship between cardiometabolic disorders, especially diabetes, and psychosis [54]. The results of this review consistently indicate that clozapine and olanzapine are associated with an increased likelihood of developing diabetes in clinical practice. The results for risperidone and quetiapine are mixed, although most studies analyzing timeto-event data indicate that both of these SGAs are associated with an increased likelihood of developing diabetes compared to FGA. Information on ziprasidone and aripiprazole is scant. Ziprasidone was consistently reported to not be associated with an increased likelihood of occurrence of diabetes in the four studies analyzing this outcome compared to patients who did not receive antipsychotics [15] and patients who received FGAs $[26,27,31]$. Only three studies reported data on the risk of diabetes with aripiprazole, showing no increased likelihood of developing diabetes compared to FGA in one study [31]; compared to antipsychoticnaïve patients, a study showed a reduced likelihood of developing diabetes with aripiprazole using a binary outcome [38], while another study showed an increased likelihood using a time-to-event outcome [39]. Head-to-head comparisons among the SGAs are limited to three studies [32-34], which indicated no difference in two of the paired comparisons $[32,33]$, including comparisons of aripiprazole with risperidone, olanzapine, quetiapine, and ziprasidone in a study using propensity scores [32]; however, a third comparison showed a slightly increased risk of occurrence of diabetes with olanzapine compared to risperidone [34].

We are not aware of any prior systematic reviews of RCTs evaluating the risk of diabetes with SGAs; however, our findings are fairly consistent with the results of systematic reviews evaluating changes in glucose levels. A recent network meta-analysis compared the effects of 18 antipsychotics on several metabolic parameters from 100 RCTs with a median treatment duration of 6 weeks [8]. The 37 studies that evaluated changes in fasting glucose indicated that clozapine and olanzapine were associated with greater increases in serum glucose concentration, while ziprasidone was associated with the lowest increase [8]. The authors did not find strong evidence of changes in serum glucose concentration with risperidone, quetiapine, ziprasidone, or aripiprazole [8]. In a previous network meta-analysis evaluating changes in glucose levels from baseline that included 47 randomized trials, the authors found that among the antipsychotics included in our review, only olanzapine was associated with a significant increase in glucose compared to placebo and only ziprasidone and risperidone were associated with significantly better results than olanzapine; when ranked using the surface under the cumulative ranking values, ziprasidone was ranked as the best and clozapine and olanzapine as the worst antipsychotics regarding their impact on serum glucose levels [55]. Yu et al. [56], in a meta-analysis of 14 studies in Chinese patients, found a higher likelihood of insulin resistance with olanzapine than with risperidone, ziprasidone, or aripiprazole. Another meta-analysis of studies conducted in healthy volunteers suggested that the occurrence of insulin resistance depends on treatment length [57], reinforcing the need for longterm studies on antipsychotic-induced metabolic disorders. 
Although the information is very limited, two studies indicated that SGAs might have a differential effect on the risk of ketoacidosis, with clozapine and olanzapine associated with an increased risk compared to FGAs, and quetiapine and risperidone showing different effects depending on age (i.e., a longer time to the occurrence of hyperglycemic emergencies [i.e., hospitalizations because of hyperglycemia, diabetic ketoacidosis, or hyperglycemic hyperosmolar state] with quetiapine compared to risperidone in elderly patients but not in younger adults). Two studies suggested that SGAs are associated with an increased likelihood of occurrence of hyperglycemic emergencies in elderly patients [51,52], regardless of whether they are treated with antidiabetics [51]. A systematic review evaluated case reports or case series of diabetic ketoacidosis with type 1 diabetes mellitus etiology, but the publication bias and uncontrolled design inherent in these reports do not allow us to assess the risk of this complication with SGAs [58]. However, it is important to stress the relevance of this potential complication associated with the use of SGAs since ketoacidosis has been associated with a high mortality rate, especially in patients with significant comorbidities and in elderly patients [59].

There is a large body of evidence based on RCTs concerning the risk of obesity or weight gain associated with SGAs [8, 57, 60]. Notably, we found only one study that evaluated obesity and two studies that evaluated weight gain. Although it is only speculative, we believe that this gap in information from population-based studies could be related to the lack of or poor information about this topic in administrative databases. Most recent meta-analyses of shortterm RCTs show that there are important differences among various SGAs in regard to the degree of weight gain or the risk of clinically relevant weight gain $[8,60,61]$. Although more limited because of the small number of studies, the results from long-term RCTs support these differences [62].

The few studies evaluating antipsychotic-induced dyslipidemia included in our review suggest that aripiprazole is not associated with an increased risk of this metabolic effect, while clozapine is, with the remaining antipsychotics showing mixed results. The results of a metaanalysis of short-term RCTs show that among the antipsychotics included in our review, risperidone, ziprasidone, and aripiprazole were not associated with deleterious effects on lipid parameters, while clozapine, olanzapine, and quetiapine were associated with increases in total cholesterol and triglycerides, and olanzapine and quetiapine were associated with increases in low density lipoprotein (LDL) cholesterol [8]. A recently published randomized short-term comparison of seven antipsychotics that included risperidone, olanzapine, quetiapine, ziprasidone, and aripiprazole showed that all of these antipsychotics were associated with significant increases in triglycerides and that risperidone, olanzapine, and quetiapine were also associated with significant increases in LDL cholesterol [63]. When evaluated as a change from baseline in the diagnosis of hyperlipidemia, ziprasidone remained unchanged, risperidone showed an increase in the proportion of patients with that diagnosis but was not statistically significant, and aripiprazole and, to a greater extent, olanzapine and quetiapine showed a statistically significant increase in the proportion of patients with hyperlipidemia at the end of the follow-up [63]. More long-term studies are needed on SGAs to further clarify their impact on lipid parameters.

Although six studies evaluated the potential association between SGAs and the occurrence of hypertension, no confirmatory evidence can be drawn from them. It appears that SGAs are associated with an increased likelihood of hypertension, and among the individual agents, in two studies, ziprasidone was associated with an almost two-fold increase in the likelihood of developing hypertension compared to FGA $[42,46]$. However, these studies of ziprasidone were conducted by the same research group using the same database and therefore these findings require replication. The limited information from randomized comparisons is not consistent. The short-term randomized head-tohead comparison of seven antipsychotics mentioned above showed no significant changes in blood pressure with any of the antipsychotics studied [63]. However, a secondary analysis of 
the 3-month results from the Clinical Antipsychotics Trials of Intervention Effectiveness (CATIE) found that in patients with baseline systolic blood pressure (SBP) above the median $(122 \mathrm{mmHg})$, all of the antipsychotics studied showed a decrease in SBP, while in patients with SBP below the median, SBP among ziprasidonetreated patients increased to a greater extent than among those treated with perphenazine $(+8.6 \mathrm{mmHg}$ vs. $+4.2 \mathrm{mmHg}, p=0.005)$ [64].

The potential determinants of the occurrence of diabetes found in our review included age, nonwhite race, obesity/BMI, hypertension, dyslipidemia, and treatment concomitant drugs such as antihypertensives, lipid-lowering drugs, and SSRIs; these factors are consistent with those reported in the literature for the general population [65-67]. However, it is important to stress that, probably because of the limitations of the information included in administrative databases, the studies included in this review did not evaluate many well-demonstrated nongenetic factors of type 2 diabetes mellitus, such as psychosocial, lifestyle, and dietary factors. Therefore, our picture of the risk factors for antipsychotic-induced diabetes is incomplete. Regarding antipsychotic treatment, several of the studies included in this review found that the risk of diabetes increases with the length of exposure. In our view, this finding is important, because among other implications, it reinforces the limitations of short-term pivotal studies on antipsychotics for establishing the metabolic profile of antipsychotics and the need for the continuous monitoring of metabolic parameters in patients who receive treatment with antipsychotics. Regarding the latter, despite the availability of recommendations for monitoring the physical health of patients with severe mental disorders, adherence to those recommendations is far from optimal [68]. Although the information on potential determinants of hyperlipidemia and hypertension is very limited, another important issue is that all of these metabolic disorders seem potential factors associated with the development of the other metabolic disorders.

In addition to the other limitations mentioned above, the literature on the metabolic adverse effects of second-generation antipsychotics has some other important limitations. The most common comparator in the studies included in this review was FGAs as a group, and the risk of metabolic side effects differs from one FGA to another. We did not find any population-based study reporting data on metabolic adverse events of long-acting injectable SGAs. Long-acting antipsychotics are associated with an increased adherence compared to oral antipsychotics [69], and consequently to a greater exposure to these drugs that in turn could be associated with a greater risk of metabolic side effects. Supporting this hypothesis, it has been reported that, compared to oral SGAs, long-acting injectable SGAs are associated with a greater risk of some adverse events, such as extrapyramidal and prolactin-related symptoms [70, 71], and some metabolic disorders, such as changes in LDL cholesterol [72]. Despite the benefits of long-acting injectable SGAs [73], it is important to know the metabolic risk associated with these formulations to facilitate the rational use of these compounds.

On the basis of the results of a meta-analysis of RCTs, the SGAs associated with better metabolic profiles are, in alphabetical order, aripiprazole, brexpiprazole, cariprazine, lurasidone, and ziprasidone [8]. However, according to our results, the worst metabolically characterized SGAs are ziprasidone and aripiprazole, and we did not find any population-based studies evaluating brexpiprazole, cariprazine, or lurasidone.

Finally, although our evaluation of the quality of the studies using the Newcastle-Ottawa scale suggests that the overall quality of the studies is fairly good, it is important to stress that only five of the studies included in this review used an adequate methodology to try to control for confounding by indication (e.g., propensity scores). Confounding by indication is a significant threat to the internal validity of observational studies of interventions [74], including the study of antipsychotic-induced metabolic disorders [75], and its potential impact was recognized by several of the authors of the studies included in this review $[11,20,22,39]$. 


\section{CONCLUSIONS}

Our results indicated that although there are relevant differences among SGAs concerning the risk of metabolic disorders, with ziprasidone and aripiprazole at one extreme of the risk continuum and olanzapine and clozapine at the other, it appears that none of the SGAs included in our review are fully devoid of these disturbances. This is a relevant finding since recent data from 14 developed countries indicates that olanzapine is the second most frequently prescribed antipsychotic in adults [76], and clozapine is increasingly prescribed [77] in addition to being the only indicated and evidence-based treatment option for treatment-resistant schizophrenia [78]. Importantly, our results suggest that the presence of a particular metabolic disorder acts as a risk factor for the occurrence of other metabolic disorders. This finding reinforces the recommendation from psychiatric clinical practice guidelines that in any patient with a metabolic disorder, it is also important to assess other contributors to metabolic syndrome [7]. In addition, as recommended in the general guidelines for the management of individual metabolic conditions in the general population, the management of these complications of antipsychotic treatment requires an integrated approach since the presence of one disorder may affect the management of the other [79-81]. However, important gaps in the routine screening of metabolic disorders are found in clinical practice [82]. The barriers associated with these gaps include healthcare system-related factors such as the fragmentation of care and time and resources constraints, and patient-related factors such as disability resulting from mental health conditions, knowledge, and skills of the patients [83].

Despite almost 20 years of real-world research using population-based studies, many gaps in the understanding of the metabolic side effects of SGAs still exist. Future research should help to characterize the metabolic profile of the increasingly used long-acting injectable antipsychotics and of new oral SGAs identified in RCTs that have a better metabolic profile, such as brexpiprazole, cariprazine, or lurasidone, and to ascertain to what extent and how antipsychotic-induced metabolic disorders contribute to the burden of disease.

\section{ACKNOWLEDGEMENTS}

The authors thank Isabel San Andrés (Incimed, Madrid, Spain) for performing the literature search

Funding. This independent research and manuscript writing and editing activities were funded by unconditional grant from Angelini Pharma España SLU. Angelini Pharma España SLU also funded the journal's rapid service fee.

Authorship. All named authors meet the International Committee of Medical Journal Editors (ICMJE) criteria for authorship for this article, take responsibility for the integrity of the work as a whole, and have given their approval for this version to be published.

Authorship Contributions. MB was responsible for the conceptualization, funding acquisition, methodology, data review, supervision and writing the draft; FR-V was responsible for the conceptualization, methodology, data extraction and writing the draft; CG-R was responsible for the conceptualization, methodology, and reviewing the draft; RR was responsible for the methodology, data extraction, and reviewing the draft; RG-H was responsible for conceptualization and reviewing the draft.

Disclosures. Miquel Bernardo has been advisory board member for Adamed, Rovi, Casen Recordati, Angelini; has received speaker honorarium from Casen, Janssen, Adamed, Menarini; has received research grants to institution from ISCIII; has received research grants from Takeda; has received consulting honorarium from Rovi; and has received travel grants from Casen Recordati. Fernando Rico-Villademoros has received consulting and/or medical writing honorarium from AstraZeneca, Casen Recordati, Janssen, Lilly, Lundbeck, Otsuka, 
Pfizer, and Sanofi; his participation in this research, including the writing of a first draft of this manuscript, has been funded by unconditional grant from Angelini Pharma España. Clemente García-Rizo has received grants from/ or served as consultant, advisor or speaker for the following entities: Adamed, Angelini, Alter, Janssen-Cilag and Lundbeck. Rosa Rojo has participated in this research funded by unconditional grant from Angelini Pharma España. Ricardo Gómez-Huelgas has no conflict of interest related with this manuscript.

Compliance with Ethics Guidelines. This article is based on previously conducted studies and does not contain any new studies with human participants or animals performed by any of the authors.

Data Availability. Data sharing is not applicable to this article as no datasets were generated or analyzed during the current study.

Open Access. This article is licensed under a Creative Commons Attribution-NonCommercial 4.0 International License, which permits any non-commercial use, sharing, adaptation, distribution and reproduction in any medium or format, as long as you give appropriate credit to the original author(s) and the source, provide a link to the Creative Commons licence, and indicate if changes were made. The images or other third party material in this article are included in the article's Creative Commons licence, unless indicated otherwise in a credit line to the material. If material is not included in the article's Creative Commons licence and your intended use is not permitted by statutory regulation or exceeds the permitted use, you will need to obtain permission directly from the copyright holder. To view a copy of this licence, visit http://creativecommons.org/licenses/by$\mathrm{nc} / 4.0 /$.

\section{REFERENCES}

1. Rosenbaum S, Stubbs B, Ward PB, Steel Z, Lederman $\mathrm{O}$, Vancampfort D. The prevalence and risk of metabolic syndrome and its components among people with posttraumatic stress disorder: a systematic review and meta-analysis. Metabolism. 2015;64:926-33.

2. Vancampfort D, Vansteelandt K, Correll CU, et al. Metabolic syndrome and metabolic abnormalities in bipolar disorder: a meta-analysis of prevalence rates and moderators. Am J Psychiatry. 2013;170: 265-74.

3. Vancampfort D, Correll CU, Wampers $\mathrm{M}$, et al. Metabolic syndrome and metabolic abnormalities in patients with major depressive disorder: a metaanalysis of prevalences and moderating variables. Psychol Med. 2014;44:2017-28.

4. Vancampfort D, Stubbs B, Mitchell AJ, et al. Risk of metabolic syndrome and its components in people with schizophrenia and related psychotic disorders, bipolar disorder and major depressive disorder: a systematic review and meta-analysis. World Psychiatry. 2015;14:339-47.

5. Vancampfort D, Hallgren M, Mugisha J, et al. The prevalence of metabolic syndrome in alcohol use disorders: a systematic review and meta-analysis. Alcohol Alcohol. 2016;51:515-21.

6. Penninx BWJH, Lange SMM. Metabolic syndrome in psychiatric patients: overview, mechanisms, and implications. Dialog Clin Neurosci. 2018;20:63-73.

7. Keepers GA, Fochtmann LJ, Anzia JM, et al. The American Psychiatric Association practice guideline for the treatment of patients with schizophrenia. Washington, DC: American Psychiatric Association; 2020.

8. Pillinger T, McCutcheon R, Vano L, et al. Comparative effects of 18 antipsychotics on metabolic function in patients with schizophrenia, predictors of metabolic dysregulation, and association with psychopathology: a systematic review and network meta-analysis. Lancet Psychiatry. 2020;7:64-77.

9. Hirsch L, Yang J, Bresee L, Jette N, Patten S, Pringsheim T. Second-generation antipsychotics and metabolic side effects: a systematic review of population-based studies. Drug Saf. 2017;40:771-81.

10. Mas S, Gassó P, Rodríguez N, et al. Personalized medicine begins with the phenotype: identifying antipsychotic response phenotypes in a first-episode psychosis cohort. Acta Psychiatr Scand. 2020;141:541-52.

11. Lund BC, Perry PJ, Brooks JM, Arndt S. Clozapine use in patients with schizophrenia and the risk of diabetes, hyperlipidemia, and hypertension: a claims-based approach. Arch Gen Psychiatry. 2001;58:1172-6. 
12. Wells G, Shea B, O'Connell D, et al. The NewcastleOttawa Scale (NOS) for assessing the quality of nonrandomised studies in meta-analyses. 2020. http://www.ohri.ca/programs/clinical_ epidemiology/oxford.asp. Accessed 20 July 2020.

13. Sharmin S, Kypri K, Khanam M, Wadolowski M, Bruno R, Mattick RP. Parental supply of alcohol in childhood and risky drinking in adolescence: systematic review and meta-analysis. Int J Environ Res Public Health. 2017;14:287.

14. Gianfrancesco FD, Grogg AL, Mahmoud RA, Wang $\mathrm{RH}$, Nasrallah HA. Differential effects of risperidone, olanzapine, clozapine, and conventional antipsychotics on type 2 diabetes: findings from a large health plan database. J Clin Psychiatry. 2002;63:920-30.

15. Gianfrancesco F, Pesa J, Wang RH, Nasrallah H. Assessment of antipsychotic-related risk of diabetes mellitus in a Medicaid psychosis population: sensitivity to study design. Am J Health Syst Pharm. 2006;63:431-41.

16. Gianfrancesco F, Wang RH, Nasrallah HA. The influence of study design on the results of pharmacoepidemiologic studies of diabetes risk with antipsychotic therapy. Ann Clin Psychiatry. 2006;18:9-17.

17. Gianfrancesco F, White R, Wang RH, Nasrallah HA. Antipsychotic-induced type 2 diabetes: evidence from a large health plan database. J Clin Psychopharmacol. 2003;23:328-35.

18. Koro CE, Fedder DO, L'Italien GJ, et al. Assessment of independent effect of olanzapine and risperidone on risk of diabetes among patients with schizophrenia: population based nested case-control study. BMJ. 2002;325:243.

19. Kornegay CJ, Vasilakis-Scaramozza C, Jick H. Incident diabetes associated with antipsychotic use in the United Kingdom general practice research database. J Clin Psychiatry. 2002;63:758-62.

20. Tu TH, Huang KL, Bai et al. Exposure to secondgeneration antipsychotics and risk of type 2 diabetes mellitus in adolescents and young adults: a nationwide study in Taiwan. J Clin Psychiatry. 2019;80:1812284.

21. Barner JC, Worchel J, Yang M. Frequency of newonset diabetes mellitus and use of antipsychotic drugs among Central Texas veterans. Pharmacotherapy. 2004;24:1529-38.

22. Citrome L, Jaffe A, Levine J, Allingham B, Robinson J. Relationship between antipsychotic medication treatment and new cases of diabetes among psychiatric inpatients. Psychiatr Serv. 2004;55: 1006-13.

23. Farwell WR, Stump TE, Wang J, Tafesse E, L'Italien G, Tierney WM. Weight gain and new onset diabetes associated with olanzapine and risperidone. J Gen Intern Med. 2004;19:1200-5.

24. Lambert BL, Chou CH, Chang KY, Tafesse E, Carson W. Antipsychotic exposure and type 2 diabetes among patients with schizophrenia: a matched case-control study of California Medicaid claims. Pharmacoepidemiol Drug Saf. 2005;14:417-25.

25. Moisan J, Turgeon M, Desjardins O, Grégoire JP. Comparative safety of antipsychotics: another look at the risk of diabetes. Can J Psychiatry. 2013;58: 218-24.

26. Guo JJ, Keck PE Jr, Corey-Lisle PK, et al. Risk of diabetes mellitus associated with atypical antipsychotic use among patients with bipolar disorder: a retrospective, population-based, case-control study. J Clin Psychiatry. 2006;67:1055-61.

27. Guo JJ, Keck PE Jr, Corey-Lisle PK, et al. Risk of diabetes mellitus associated with atypical antipsychotic use among Medicaid patients with bipolar disorder: a nested case-control study. Pharmacotherapy. 2007;27:27-35.

28. Lambert BL, Cunningham FE, Miller DR, Dalack GW, Hur K. Diabetes risk associated with use of olanzapine, quetiapine, and risperidone in veterans health administration patients with schizophrenia. Am J Epidemiol. 2006;164:672-81.

29. Leslie DL, Rosenheck RA. Incidence of newly diagnosed diabetes attributable to atypical antipsychotic medications. Am J Psychiatry. 2004;161: 1709-11.

30. Miller EA, Leslie DL, Rosenheck RA. Incidence of new-onset diabetes mellitus among patients receiving atypical neuroleptics in the treatment of mental illness: evidence from a privately insured population. J Nerv Ment Dis. 2005;193:387-95.

31. Yood MU, DeLorenze G, Quesenberry CP Jr, et al. The incidence of diabetes in atypical antipsychotic users differs according to agent-results from a multisite epidemiologic study. Pharmacoepidemiol Drug Saf. 2009;18:791-9.

32. Citrome L, Collins JM, Nordstrom BL, et al. Incidence of cardiovascular outcomes and diabetes mellitus among users of second-generation antipsychotics. J Clin Psychiatry. 2013;74: 1199-206.

33. Ollendorf DA, Joyce AT, Rucker M. Rate of newonset diabetes among patients treated with atypical 
or conventional antipsychotic medications for schizophrenia. MedGenMed. 2004;6:5.

34. Caro JJ, Ward A, Levinton C, Robinson K. The risk of diabetes during olanzapine use compared with risperidone use: a retrospective database analysis. J Clin Psychiatry. 2002;63:1135-9.

35. Hayes JF, Marston L, Walters K, Geddes JR, King M, Osborn DP. Adverse renal, endocrine, hepatic, and metabolic events during maintenance mood stabilizer treatment for bipolar disorder: a populationbased cohort study. PLoS Med. 2016;13:e1002058.

36. Liao $\mathrm{CH}$, Chang CS, Wei WC, et al. Schizophrenia patients at higher risk of diabetes, hypertension and hyperlipidemia: a population-based study. Schizophr Res. 2011;126:110-6.

37. Lin CY, Wu YH, Wang HS, Chen PK, Lin YF, Chien IC. Risk of new onset type II DM in MDD patients receiving second-generation antipsychotics treatment: a nationwide cohort study. Depress Anxiety. 2016;33:435-43.

38. Nielsen J, Skadhede S, Correll CU. Antipsychotics associated with the development of type 2 diabetes in antipsychotic-naïve schizophrenia patients. Neuropsychopharmacology. 2010;35:1997-2004.

39. Rajkumar AP, Horsdal HT, Wimberley $\mathrm{T}$, et al. Endogenous and antipsychotic-related risks for diabetes mellitus in young people with schizophrenia: a Danish population-based cohort study. Am J Psychiatry. 2017;174:686-94.

40. Stroup TS, Gerhard T, Crystal S, Huang C, Olfson M. Comparative effectiveness of clozapine and standard antipsychotic treatment in adults with schizophrenia. Am J Psychiatry. 2016;173:166-73.

41. Lipscombe LL, Austin PC, Alessi-Severini S, et al. Atypical antipsychotics and hyperglycemic emergencies: multicentre, retrospective cohort study of administrative data. Schizophr Res. 2014;154: 54-60.

42. Jerrell JM, McIntyre RS, Tripathi A. Incidence and costs of cardiometabolic conditions in patients with schizophrenia treated with antipsychotic medications. Clin Schizophr Relat Psychoses. 2010;4: 161-8.

43. Hsu JH, Chien IC, Lin CH, Chou YJ, Chou P. Hyperlipidemia in patients with schizophrenia: a national population-based study. Gen Hosp Psychiatry. 2012;34:360-7.

44. Lambert BL, Chang KY, Tafesse E, Carson W. Association between antipsychotic treatment and hyperlipidemia among California Medicaid patients with schizophrenia. J Clin Psychopharmacol. 2005;25:12-8.

45. Olfson M, Marcus SC, Corey-Lisle P, Tuomari AV, Hines P, L'Italien GJ. Hyperlipidemia following treatment with antipsychotic medications. Am J Psychiatry. 2006;163:1821-5.

46. Jerrell JM, McIntyre RS. Cerebro- and cardiovascular conditions in adults with schizophrenia treated with antipsychotic medications. Hum Psychopharmacol. 2007;22:361-4.

47. Wu EL, Chien IC, Lin CH, Chou YJ, Chou P. Increased risk of hypertension in patients with major depressive disorder: a population-based study. J Psychosom Res. 2012;73:169-74.

48. Erickson SC, Le L, Zakharyan A, et al. New-onset treatment-dependent diabetes mellitus and hyperlipidemia associated with atypical antipsychotic use in older adults without schizophrenia or bipolar disorder. J Am Geriatr Soc. 2012;60:474-9.

49. Jalbert JJ, Daiello LA, Eaton CB, Miller SC, Lapane KL. Antipsychotic use and the risk of diabetes in nursing home residents with dementia. Am J Geriatr Pharmacother. 2011;9:153-63.

50. Kisely S, Cox M, Campbell LA, Cooke C, Gardner D. An epidemiologic study of psychotropic medication and obesity-related chronic illnesses in older psychiatric patients. Can J Psychiatry. 2009;54:269-74.

51. Lipscombe LL, Lévesque L, Gruneir A, et al. Antipsychotic drugs and hyperglycemia in older patients with diabetes. Arch Intern Med. 2009;169: 1282-9.

52. Lipscombe LL, Lévesque LE, Gruneir A, et al. Antipsychotic drugs and the risk of hyperglycemia in older adults without diabetes: a populationbased observational study. Am J Geriatr Psychiatry. 2011;19:1026-33.

53. Stroup TS, Geddes JR. Randomized controlled trials for schizophrenia: study designs targeted to distinct goals. Schizophr Bull. 2008;34:266-74.

54. Garcia-Rizo C, Fernandez-Egea E, Bernardo M, Kirkpatrick B. The thrifty psychiatric phenotype. Acta Psychiatr Scand. 2015;131:18-20.

55. Zhang Y, Liu Y, Su Y, et al. The metabolic side effects of 12 antipsychotic drugs used for the treatment of schizophrenia on glucose: a network meta-analysis. BMC Psychiatry. 2017;17:373.

56. Yu L, Wu S, Deng Y, Lei J, Yu L, Li W. Insulin resistance induced by olanzapine and other secondgeneration antipsychotics in Chinese patients with 
schizophrenia: a comparative review and metaanalysis. Eur J Clin Pharmacol. 2019;75:1621-9.

57. Burghardt KJ, Seyoum B, Mallisho A, Burghardt PR, Kowluru RA, Yi Z. Atypical antipsychotics, insulin resistance and weight; a meta-analysis of healthy volunteer studies. Prog Neuropsychopharmacol Biol Psychiatry. 2018;83:55-63.

58. Polcwiartek C, Vang $\mathrm{T}$, Bruhn $\mathrm{CH}$, Hashemi N, Rosenzweig M, Nielsen J. Diabetic ketoacidosis in patients exposed to antipsychotics: a systematic literature review and analysis of Danish adverse drug event reports. Psychopharmacology. 2016;233:3663-72.

59. French EK, Donihi AC, Korytkowski MT. Diabetic ketoacidosis and hyperosmolar hyperglycemic syndrome: review of acute decompensated diabetes in adult patients. BMJ. 2019;365:11114.

60. Barton BB, Segger F, Fischer K, Obermeier M, Musil R. Update on weight-gain caused by antipsychotics: a systematic review and meta-analysis. Expert Opin Drug Saf. 2020;19:295-314.

61. Huhn M, Nikolakopoulou A, Schneider-Thoma J, et al. Comparative efficacy and tolerability of 32 oral antipsychotics for the acute treatment of adults with multi-episode schizophrenia: a systematic review and network meta-analysis. Lancet. 2019;394:939-51.

62. Kishimoto T, Hagi K, Nitta M, Kane JM, Correll CU. Long-term effectiveness of oral second-generation antipsychotics in patients with schizophrenia and related disorders: a systematic review and metaanalysis of direct head-to-head comparisons. World Psychiatry. 2019;18:208-24.

63. Zhang Y, Wang Q, Reynolds GP, et al. Metabolic effects of 7 antipsychotics on patients with schizophrenia: a short-term, randomized, open-label, multicenter, pharmacologic trial. J Clin Psychiatry. 2020;81:19m12785.

64. Meyer JM, Davis VG, Goff DC, et al. Change in metabolic syndrome parameters with antipsychotic treatment in the CATIE schizophrenia trial: prospective data from phase 1 . Schizophr Res. 2008;101:273-86.

65. Bellou V, Belbasis L, Tzoulaki I, Evangelou E. Risk factors for type 2 diabetes mellitus: an exposurewide umbrella review of meta-analyses. PLoS One. 2018;13:e0194127.

66. Fathallah N, Slim R, Larif S, Hmouda H, Ben SC. Drug-induced hyperglycaemia and diabetes. Drug Saf. 2015;38:1153-68.
67. Haffner SM. Epidemiology of type 2 diabetes: risk factors. Diabetes Care. 1998;21:C3-6.

68. Mangurian C, Schillinger D, Newcomer JW, et al. Diabetes screening among antipsychotic-treated adults with severe mental illness in an integrated delivery system: a retrospective cohort study. J Gen Intern Med. 2018;33:79-86.

69. Suzuki T. A further consideration on long-acting injectable versus oral antipsychotics in the treatment of schizophrenia: a narrative review and critical appraisal. Expert Opin Drug Deliv. 2016;13: 253-64.

70. Kishi T, Oya K, Iwata N. Long-acting injectable antipsychotics for prevention of relapse in bipolar disorder: a systematic review and meta-analyses of randomized controlled trials. Int J Neuropsychopharmacol. 2016;19:pyw038.

71. Park SC, Choi MY, Choi J, et al. Comparative efficacy and safety of long-acting injectable and oral second-generation antipsychotics for the treatment of schizophrenia: a systematic review and metaanalysis. Clin Psychopharmacol Neurosci. 2018;16: 361-75.

72. Misawa F, Kishimoto T, Hagi K, Kane JM, Correll CU. Safety and tolerability of long-acting injectable versus oral antipsychotics: a meta-analysis of randomized controlled studies comparing the same antipsychotics. Schizophr Res. 2016;176: 220-30.

73. Taipale H, Mittendorfer-Rutz E, Alexanderson K, et al. Antipsychotics and mortality in a nationwide cohort of 29,823 patients with schizophrenia. Schizophr Res. 2018;197:274-80.

74. Walker AM, Stampfer MJ. Observational studies of drug safety. Lancet. 1996;348:489.

75. Rico-Villademoros F, Calandre EP. Risk of bias in observational studies of interventions: the case of antipsychotic-induced diabetes. Lancet Psychiatry. 2016;3:103-4.

76. Hálfdánarson Ó, Zoëga H, Aagaard L, et al. International trends in antipsychotic use: a study in 16 countries, 2005-2014. Eur Neuropsychopharmacol. 2017;27:1064-76.

77. Bachmann CJ, Aagaard L, Bernardo M, et al. International trends in clozapine use: a study in 17 countries. Acta Psychiatr Scand. 2017;136:37-51.

78. Kane JM, Agid O, Baldwin ML, et al. Clinical guidance on the identification and management of treatment-resistant schizophrenia. J Clin Psychiatry. 2019;80:18com 12123 . 
79. Buse JB, Wexler DJ, Tsapas A, et al. 2019 Update to: management of hyperglycemia in type 2 diabetes, 2018. A consensus report by the American Diabetes Association (ADA) and the European Association for the Study of Diabetes (EASD). Diabetes Care. 2020;43:487-93.

80. Mach F, Baigent C, Catapano AL, et al. 2019 ESC/ EAS Guidelines for the management of dyslipidaemias: lipid modification to reduce cardiovascular risk. Eur Heart J. 2020;41:111-88.

81. Williams B, Mancia G, Spiering W, et al. 2018 ESC/ ESH guidelines for the management of arterial hypertension: the task force for the management of arterial hypertension of the European Society of
Cardiology and the European Society of Hypertension: the task force for the management of arterial hypertension of the European Society of Cardiology and the European Society of Hypertension. J Hypertens. 2018;36:1953-2041.

82. Morrato EH, Campagna EJ, Brewer SE, et al. Metabolic testing for adults in a state Medicaid program receiving antipsychotics: remaining barriers to achieving population health prevention goals. JAMA Psychiatry. 2016;73:721-30.

83. Ali RA, Jalal Z, Paudyal V. Barriers to monitoring and management of cardiovascular and metabolic health of patients prescribed antipsychotic drugs: a systematic review. BMC Psychiatry. 2020;20:581. 